\title{
XADREZ, DO REAL AO VIRTUAL
}

\author{
José Francisco de Magalhães Netto* \\ Orivaldo Lira Tavares** \\ Crediné Silva de Menezes ${ }^{* * *}$
}

\begin{abstract}
Resumo: Este artigo propõe o AVAX, um ambiente virtual de prática e aprendizagem de Xadrez, baseado na tecnologia de sistemas multiagentes. No AVAX, a comunidade composta por usuários e agentes heterogêneos, forma o que designamos de Comunidade Virtual de Convivência. A realização do AVAX é baseada na FIPA e o ambiente de implementação escolhido é o JADE. Para avaliar o sistema apresentamos uma proposta preliminar, onde se atribuem valores às facilidades encontradas em ambientes típicos para a prática do Xadrez. Palavras-chaves: ambiente virtual de aprendizagem, sistemas multiagentes, jogos educacionais, xadrez.
\end{abstract}

Abstract: This article proposes the AVAX, a virtual environment of practice and learning of Chess, based in the technology of multiagent systems. In the AVAX, the composed community by users and heterogeneous agents, form what do we assign of Virtual Community of Convivence. The achievement of the AVAX is based in the FIPA and the environment of implementation choosen is the JADE. For it evaluate the system we present a preliminary proposal, where attribute themselves values to the facilities found in typical environments for the practical one of the Chess.

Key-words: learning virtual environment, multiagent systems, educative games, chess.

\section{Introdução}

Com a disseminação da Web, diversos ambientes que apóiam comunidades virtuais têm surgido. Atualmente observa-se a existência de ambientes virtuais que provêem facilidades aos usuários para a realização de seus objetivos específicos, mais direcionados aos interesses do grupo. Com isso têm-se, além das ferramentas clássicas disponíveis nesses ambientes (ferramentas de email, fóruns, chats, murais etc), outras ferramentas que visam apoiar as interações sociais dentro do grupo e/ou ferramentas que desempenham tarefas próprias da comunidade.

Usando o paradigma de Sistemas Multiagentes, empregando o conceito de Comunidade Virtual de Convivência, desenvolvemos uma arquitetura para o AmCorA (AmCorA, 2005), um ambiente virtual de aprendizagem que serve de plataforma para apoiar comunidades virtuais, já utilizado em diversos experimentos (Menezes et al., 1999) (Menezes et al., 2003). Com o intuito de explorar o potencial desta arquitetura desenvolveu-se o sistema AVAX (Ambiente Virtual de Aprendizagem de Xadrez).

A escolha do tema - Xadrez - deve-se a diversos fatores e entre os principais citam-se: pela possibilidade de se construir um software educacional (Netto, 1995) para um uso coletivo; por ser possível importar objetos de aprendizagem, tais como programas, partidas, estudos e conhecimento heurístico, disponíveis em uma ampla literatura e através da Web (Netto et al., 2005a); pelos relatos de experiências usando esse jogo em ambientes virtuais (Direne e Schafer 2000) (Direne et al. 2004); e pela existência, em nossas Universidades, de uma comunidade interessada em participar de eventos e sugerir melhorias. Além destes fatores, o jogo de Xadrez desperta grande interesse pelo seu lado esportivo e por ser reconhecido como estimulador de desenvolvimento de capacidades cognitivas (Netto et al., 1995) (Netto et al., 2005a), tendo sua prática incentivada em escolas por meio de abordagens tradicionais (por exemplo: grupos presenciais que se reúnem, jogam e treinam em clubes) como também por meio da integração com ferramentas computacionais já existentes (Direne et al., 2004).

\footnotetext{
"Departamento de Ciência da Computação - UFAM, Programa de Pós-graduação em Engenharia Elétrica - UFES, inetto@dcc.fua.br

*** Programa de Pós-graduação em Informática - UFES, tavares@inf.ufes.br

*** Programa de Pós-graduação em Engenharia Elétrica - UFES, Programa de Pós-graduação em Informática na Educação - UFES, credine@inf.ufes.br 
O propósito do AVAX é inovar o suporte à interação nas comunidades virtuais de Xadrez utilizando-se de recursos de Recuperação de Informações e de Sistemas Multiagentes. Propomos um ambiente onde usuários de variados níveis de jogo possam desempenhar as atividades básicas do Xadrez - jogar, participar de competições, analisar partidas e treinar. $\mathrm{O}$ presente texto trata do processo de desenvolvimento deste sistema.

O restante do artigo está organizado da seguinte maneira: a seção 2 apresenta os trabalhos correlatos; a seção 3 apresenta a arquitetura que é utilizada; a seção 4 discute uma realização para o AVAX e na seção 5 apresentamos uma proposta de avaliação para o AVAX. O artigo é finalizado com a seção 6, onde estão as conclusões e os trabalhos futuros.

\section{Trabalhos Correlatos}

São apresentados nesta seção os trabalhos correlatos a softwares de treinamento de Xadrez (seção 2.1) e a Ambientes de Apoio a Aprendizagem Virtual (seção 2.2). Na seção 2.3 faz-se um relato das contribuições desses trabalhos na concepção do projeto.

\subsection{Treinamento em Xadrez por Meio de Software}

Estão disponíveis vários softwares e ambientes para a prática de Xadrez. Escolheu-se dois softwares por serem representativos das principais características atuais de treinamento de Xadrez para iniciantes usando computador: ICC (ICC, 2005) e Chessmaster (Chessmaster, 2005). Estes dois softwares estão ente os mais utilizados, ao lado do ChessBase, que é utilizado principalmente por jogadores experientes.

O ICC (Internet Chess Club) dá opção aos seus usuários de jogarem on-line e observarem os eventos que acontecem no ambiente.

O ICC é um dos sistemas mais usados quando se trata de jogar partidas de Xadrez via Web. O jogador ao se conectar no ICC tem acesso a uma lista de jogadores conectados, composta de seus respectivos nomes, status (livre, ocupado etc) e força de jogo (rating), e, então, escolhe um usuário disponível e pode convidá-lo para jogar uma partida. $\mathrm{O}$ jogador visualiza um tabuleiro onde são registrados os lances realizados durante a partida, bem como o tempo restante disponível uma vez que as partidas são jogadas em um ritmo previamente combinado. Uma grande comunidade acaba se formando dentro do ICC composta de jogadores de vários níveis e nacionalidades, que além de jogar Xadrez, trocam informações sobre os eventos enxadrísticos e jogadores.

O Chessmaster possui um grande volume de informações (partidas, testes, posições para análise, aulas em vídeos etc). Jogando em sua força máxima o Chessmaster é um forte jogador, com rating superior ao de muitos Grandes Mestres Internacionais. $\mathrm{O}$ sistema possui algumas características que facilitam a aprendizagem para jogadores iniciantes, como, por exemplo, emitir um aviso quando o jogador realiza uma jogada muito fraca no tabuleiro. O sistema avalia a força de jogo do novato através de uma série de partidas. O jogador pode configurar o tabuleiro em diversas opções de 2D e 3D. O usuário pode configurar o estilo de jogo do Chessmaster, procurando tornálo semelhante ao estilo de um ex-campeão mundial famoso. Dotado de uma interface moderna, grande força de jogo, e possibilitando configurações por parte do usuário, $\mathrm{o}$ Chessmaster é extremamente atraente, especialmente para jogadores iniciantes.

\subsection{Sistemas de Apoio a Aprendizagem Virtual}

O I-Help é um sistema de ajuda a pares (peer-help system) baseado na Internet desenvolvido na Universidade de Saskatchewan, tendo sido utilizado em vários experimentos, envolvendo milhares de usuários. O sistema trabalha localizando recursos 
materiais nas páginas da Web, em fóruns de discussão, etc e pessoas dispostas a ajudar a partir de uma requisição de um usuário (Vassileva et al., 2001) (Vassileva, 2003).

O I-Help é baseado em uma arquitetura multiagente consistindo de agentes pessoais e agentes de aplicações, que se referem às ferramentas disponíveis (Vassileva et al., 2001). Os agentes usam uma ontologia em comum e os usuários são representados no sistema através de um modelo do aprendiz, descrito mais detalhadamente em (Bull, 2001). Várias versões do I-Help foram elaboradas usando esquemas de comunicação distintos como KQML e Corba, desde a primeira implementação totalmente distribuída até a versão atual centralizada (Bull et al., 2001) (Deters, 2005) (Vassileva et al., 2001).

O projeto PEDANT (Pedagogical Agents For Modeling On-Line And Computer-Interactive Learning), desenvolvido na Universidade de Melbourne, tem como objetivo "investigar a relação entre a maneira com que estudantes usam recursos interativos educacionais on-line e a qualidade de sua experiência educacional" (Markham, 2005). O cerne da proposta é a geração de agentes de software que monitoram as atividades de estudantes no sistema e eventualmente realizam análises. Dentro destes agentes, há agentes que registram as ações em logs e agentes que transformam informações contidas nestes logs (Information Log) para um nível maior de abstração (Knowledge Log).

O Projeto JADE (Java Agent Framework for Distance Learning Environments) propõe uma infra-estrutura para o projeto, desenvolvimento e implementação de Ambientes Inteligentes de Aprendizagem Distribuída (Silveira e Vicari, 2002). O JADE é baseado no paradigma de Sistemas Multiagentes, compondo-se de agentes responsáveis por cada estratégia de ensino, de um agente responsável pelo controle geral do sistema e de um Gerenciador de Comunicação. Os agentes trocam mensagens no formato KQML.

\subsection{Contribuições dos Trabalhos Correlatos ao Projeto}

Incorporou-se ao projeto elementos de interface dos softwares avaliados. No treinamento em Xadrez por meio de software observa-se que em sistemas como o Chessmaster as interações não levam em consideração o perfil e o histórico do usuário. Algumas configurações que são feitas pelo usuário poderiam ser feitas automaticamente pelo sistema, com a finalidade de dinamizar a aprendizagem e a satisfação do usuário. Em ambientes como o ICC observa-se que a questão central é a busca de oponentes e a formação de grupos. Uma sugestão para dinamizar este processo é dotar o ambiente de ferramentas que facilitem a formação de pares de jogadores e de grupos, usando critérios como as preferências dos jogadores envolvidos, o tipo de tarefa a ser desempenhada (partida simples, torneio, análise), a força de jogo, os históricos dos jogadores, as disponibilidades etc.

Os pontos destacados nos Sistemas de Apoio a Aprendizagem Virtual foram: o convívio de agentes pessoais e de agentes de aplicação no I-Help; o registro das ações de usuários e, em particular, o uso de logs como instrumento de registro de ações dos usuários em (Markham, 2005); a estrutura distribuída dos agentes no JADE e o uso de KQML (Silveira e Vicari, 2002); o uso de ontologias, em I-Help. Notou-se o uso de arquiteturas centradas em ontologias, no uso cada vez mais freqüente de perfis de usuários para potencializar as interações, no amplo uso de tecnologias de representação baseadas em XML; e na utilização de padrões de comunicação como o KQML, e mais recentemente o FIPA (FIPA 2005) em projetos dessa natureza. 


\section{Arquitetura Geral de um Ambiente de Convivência}

No contexto deste trabalho considera-se que um Ambiente Virtual de Convivência é um ambiente que apóia as interações de agentes heterogêneos (pessoas, software e máquinas), possibilitando a realização de tarefas em comum. Para se criar este ambiente, cada usuário é representado no sistema por um agente pessoal inteligente denominado clone. Inicialmente o clone captura o perfil do usuário que ele representa e nas etapas seguintes o aprendizado do clone baseia-se no registro e análise das interações do seu dono nas diversas sessões com a comunidade e nas interações dos demais agentes (reais e virtuais).

Os agentes compartilham uma linguagem e uma ontologia comuns. A linguagem é baseada no padrão ACL FIPA, permitindo extensões em XML (FIPA, 2005). A ontologia possibilita que os conceitos usados pelos diversos agentes tenham $o$ mesmo significado.

A proposta de uma arquitetura é mostrada na Figura 1. A arquitetura caracterizase, essencialmente, por ser uma comunidade de agentes chamados clones, pelo uso de agentes buscadores de provedores de serviços e pelo uso da linguagem de comunicação de agentes ACL FIPA e de uma ontologia em comum (Netto et al., 2004).

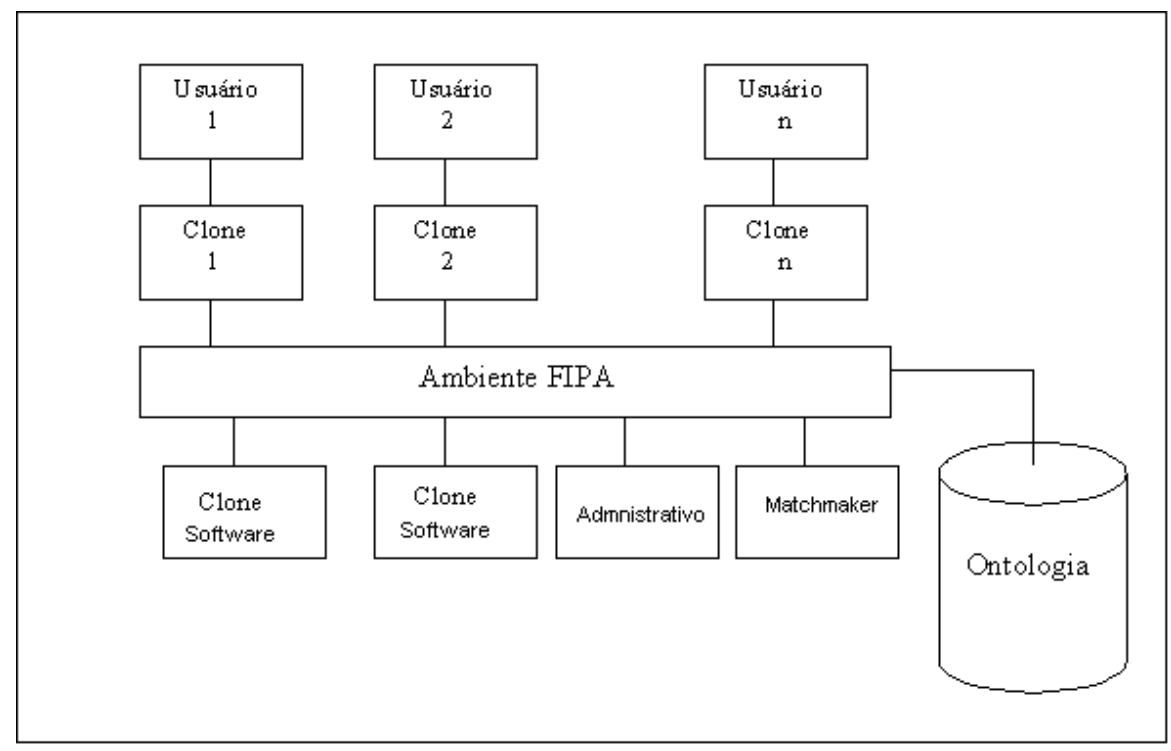

Figura 1. Arquitetura Multiagente para o AmCorA (Netto et al., 2004)

Os agentes básicos desta arquitetura são: Clone, Matchmaker e Administrativo. O Clone é um agente virtual que representa um usuário, um software ou um equipamento. O Matchmaker é um agente ou uma comunidade de agentes (agência) que provê o serviço de busca de provedores para um determinado serviço. É composto de uma tabela de serviços mais freqüentemente acessados (Páginas Amarelas) e de uma relação geral de serviços (Páginas Brancas). O Administrativo é uma comunidade de agentes que auxiliam os demais agentes a realizarem uma tarefa.

\section{O AVAX}

Compõem o cenário para o qual o sistema é projetado, jogadores de Xadrez geograficamente distribuídos e conectados em rede, desenvolvendo atividades diversas como jogos e disputas de torneios, treinamentos, análise de partidas, resolução de problemas, ou simplesmente observando o desenrolar das atividades. Nesta comunidade os jogadores são de diversos níveis, desde principiantes até jogadores experientes. Os participantes têm o perfil de jogadores e alguns também têm o perfil adicional de 
técnico, professor ou organizador de eventos. Participam também da comunidade, agentes de software com diversas competências, que vão desde a capacidade de jogar contra oponentes humanos até agentes capazes de analisar partidas ou situações de jogo em particular (por exemplo: análise de finais de partidas).

O passo inicial foi criar uma ontologia do contexto Aprendizagem de Xadrez, com a finalidade de inteirar-se dos conceitos relacionados à área e servir de base para o projeto do sistema. Os conceitos e suas relações foram organizados sob a forma de um mapa conceitual, que é apresentado na Figura 2.

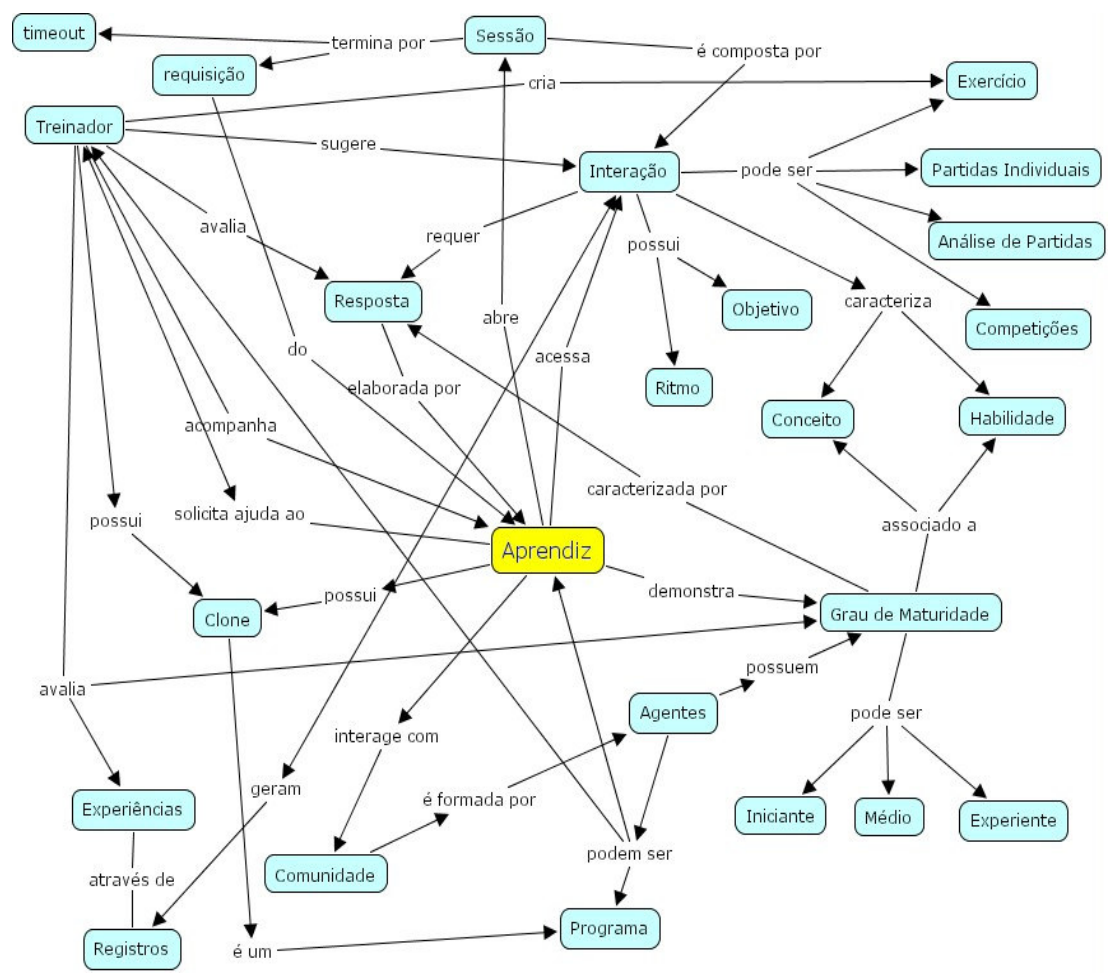

Figura 2. Ontologia de domínio da Aprendizagem em Xadrez

O passo subseqüente foi definir estratégia de interação entre os agentes, elemento central na concepção pois define o mecanismo de aprendizagem. No Xadrez a aprendizagem dá-se por sucessivas interações do aprendiz com jogadores mais experientes, onde são analisadas em conjunto posições e partidas e discutidos conceitos, técnicas e jogadas mais avançadas (Netto et al., 2005b). O processo de aprendizagem é denominado treinamento pelos praticantes deste jogo. Os treinamentos são conduzidos de forma gradual, apresentando desde situações básicas até situações de maior complexidade. O treinamento em Xadrez pode ser conduzido usando a abordagem da Zona Proximal de Desenvolvimento (Netto, 1995), proposto por Vigotsky, como sendo a área em que indivíduos solucionam problemas com a cooperação de pares mais experientes (Vigotsky, 1998).

Na figura 3 apresenta-se os casos de uso do sistema. 


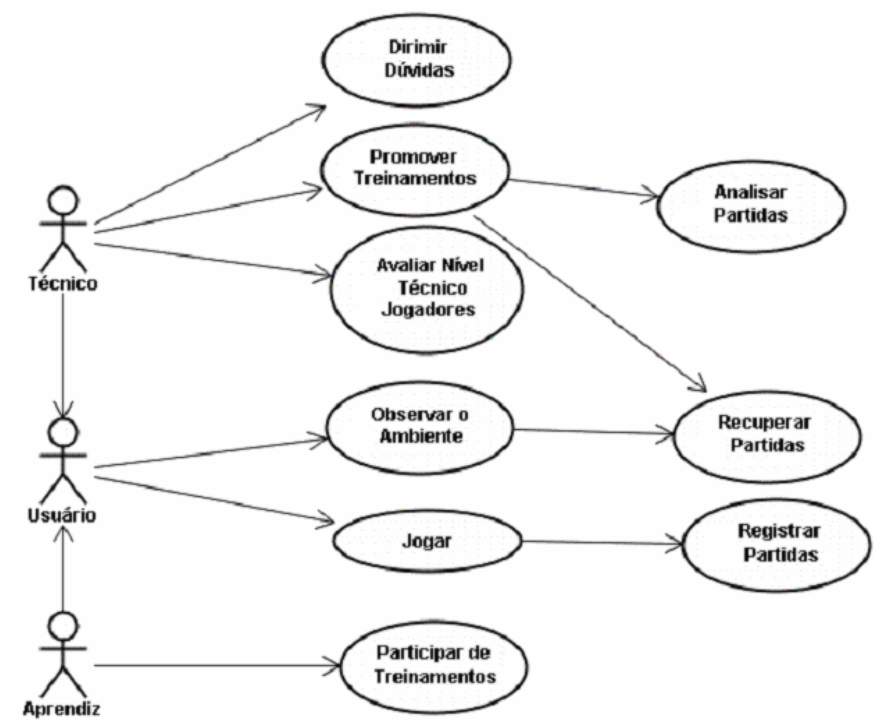

Figura 3. Casos de uso do AVAX

O usuário ao se cadastrar no sistema tem o seu perfil caracterizado pelo nome, idade, sexo e com a avaliação aproximada de sua força de jogo (rating, na terminologia enxadrística) (Netto et al., 2005a) (Netto et al., 2005b). É criado um agente chamado clone para cada usuário, cuja função é aprender sobre seu proprietário e substituí-lo em algumas situações (Netto et al., 2004). Agentes denominados Matchmakers realizam a busca de agentes que possam atender a uma requisição de um usuário (Netto et al., 2004). O sistema é composto, também, por inúmeros agentes especialistas em Xadrez. Denomina-se Ambiente Virtual de Convivência ao conjunto de pessoas, clones e agentes em geral (Netto et al., 2004). Agentes monitoram constantemente os progressos do usuário, registrando suas ações e atualizando seu rating. As constantes atualizações de força de jogo e histórico propiciam que as interações sejam direcionadas para serem mais apropriadas para o perfil do jogador. A comunidade de agentes coopera com as pessoas quando são solicitados ou de forma autônoma, na solução dos diversos problemas que surgem (Netto et al., 2004) (Netto et al., 2005a). No sistema estão cadastradas centenas de posições de estudo, cada uma associada a conceitos e habilidades do Xadrez. As posições e partidas são mostradas para o usuário e suas respostas são analisadas, inferindo-se o grau de domínio do usuário sobre o tema em discussão.

Quando um Aprendiz solicita ajuda, o Treinador procura caracterizar o tipo de pedido, freqüentemente uma dúvida relacionada a uma das fases do jogo de Xadrez abertura, meio-jogo ou final. O Treinador procura também caracterizar o grau de maturidade do Aprendiz. Com estas informações, o Treinador procura por meio de consultas a sua base de conhecimentos que é freqüentemente atualizada com o nome dos agentes e suas competências, identificar na comunidade os agentes capazes de realizar a ajuda. $\mathrm{O}$ Treinador entra em contato com o agente selecionado e caso este esteja disponível, direciona sua resposta para o Aprendiz. Caso o agente escolhido não esteja disponível, a solicitação é direcionada para outro agente, até que se consiga um agente disponível.

Na Figura 4 apresenta-se uma interação entre o AVAX e um usuário. 


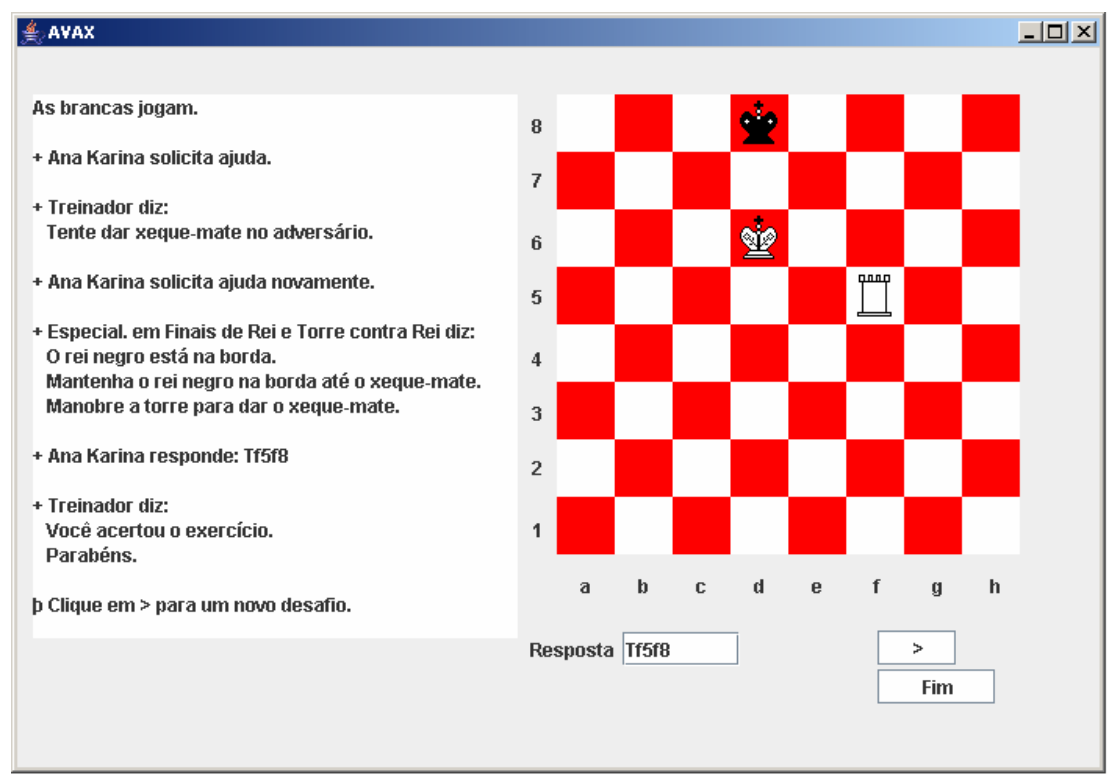

Figura 4. Treinando Xadrez usando o AVAX

Os agentes trocam mensagens, promovendo a cooperação. A Figura 5 apresenta uma simulação em que um agente solicita ajuda ao Matchmaker e este direciona o pedido ao agente especialista no tipo de posição. O agente especialista retorna uma resposta ao agente que fez a requisição inicial. O Matchmaker é atualizado freqüentemente com os nomes de agentes e habilidades, podendo assim indicar agentes que possam satisfazer a uma determinada requisição. A visualização das trocas de mensagens entre agentes no JADE (Java Agent Development Framework) é feita por meio da ferramenta Sniffer (JADE, 2005).

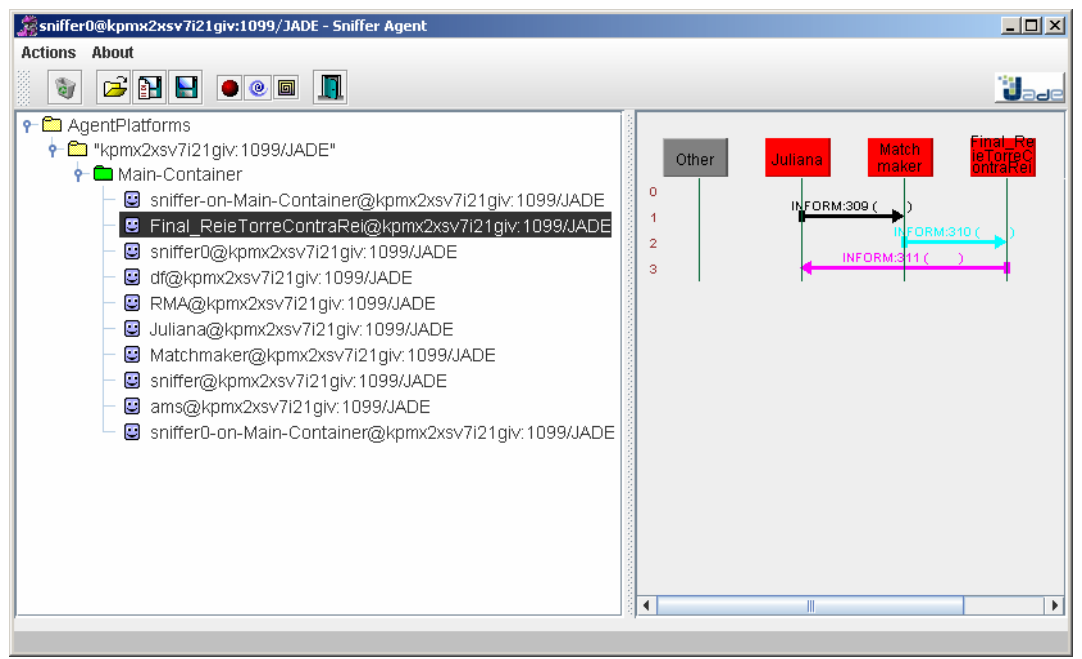

Figura 5. Troca de mensagens entre Agentes no AVAX

O ambiente AVAX incorpora uma base de conhecimento que inclui classificações de aberturas e variantes, conhecimentos sobre finais, posições de estudo e partidas capturadas em sítios, em bancos de dados de acesso público e em livros clássicos. O protótipo AVAX é desenvolvido usando-se Java 2 Platform Standard Edition (J2SE) 5.0 (J2SE, 2005) e o banco de dados MySQL (MySQL, 2005) como camada de persistência. A versão do AVAX a ser disponibilizada através da Web utiliza 
as tecnologias servlet e JSP (JavaServer Pages) (JSP, 2005) e a plataforma JADE (Java Agent DEvelopment Framework) como integradora dos agentes (JADE, 2005).

\section{Comparação da Abordagem utilizada no AVAX com outras abordagens.}

Pretende-se avaliar as contribuições introduzidas pelo AVAX para comunidades de enxadristas. Com esta intenção esboçamos uma proposta que consiste em atribuir valor, em alguns ambientes, às facilidades desejáveis em ambientes desta natureza.

Selecionamos alguns ambientes típicos utilizados para a prática do enxadrismo e destacamos os elementos de interação (jogar, treinar e observar), são eles: (I) o jogador desloca-se a um clube ou a outro local para participar dos eventos; (II) o jogador aciona um computador, sem acesso à Internet e ativa um programa específico que joga Xadrez; (III) o jogador acessa um computador, conectado à Internet e navega à procura de parceiros e busca de demais informações; e (IV) o jogador aciona um computador, com acesso à Internet e com acesso ao ambiente de agentes do AVAX. A tabela 1 mostra os componentes, interações, mídias e suporte computacional associados a cada um dos ambientes descritos.

Tabela 1. Tipo, Componentes e Interações, Mídias e Suporte Computacional dos Ambientes.

\begin{tabular}{|l|l|l|l|l|}
\hline Tipo & Componentes & Interações & Mídias & $\begin{array}{l}\text { Suporte } \\
\text { Computacional }\end{array}$ \\
\hline I & Pessoas & Pessoa-Pessoa & $\begin{array}{l}\text { Papel (livros, planilhas } \\
\text { impressas) }\end{array}$ & Nenhum \\
\hline II & $\begin{array}{l}\text { Pessoas, } \\
\text { Programas }\end{array}$ & $\begin{array}{l}\text { Pessoa- } \\
\text { Programa }\end{array}$ & Digital (Arquivos) & $\begin{array}{l}\text { Computador, } \\
\text { Programa específico }\end{array}$ \\
\hline III & $\begin{array}{l}\text { Pessoas, } \\
\text { Programas }\end{array}$ & $\begin{array}{l}\text { Pessoa- } \\
\text { Programa } \\
\text { Pessoa-Pessoa }\end{array}$ & Digital (Arquivos) & $\begin{array}{l}\text { Computador, } \\
\text { Acesso à Internet }\end{array}$ \\
\hline IV & $\begin{array}{l}\text { Pessoas, } \\
\text { Agentes }\end{array}$ & $\begin{array}{l}\text { Pessoa-Pessoa } \\
\text { Pessoa-Agente } \\
\text { Agente-Pessoa } \\
\text { Agente- } \\
\text { Agente }\end{array}$ & Digital (Arquivos) & $\begin{array}{l}\text { Computador, } \\
\text { Acesso à Internet, } \\
\text { Banco de dados, } \\
\text { Agentes }\end{array}$ \\
\hline
\end{tabular}

A tabela 2 mostra as ações mais freqüentes praticadas por usuários em ambientes de Xadrez e suas avaliações em relação aos ambientes anteriormente citados. Usamos uma pontuação de 0 a 4 , onde 0 significa a ausência do fator e 4 a presença enfática do fator. Às situações intermediárias de intensidade da presença do fator atribuímos os valores 1,2 e 3 . 
Tabela 2. Aç̃es e Avaliações correspondentes a cada Ambiente

\begin{tabular}{|l|l|l|l|l|}
\hline Ações & I & II & III & IV \\
\hline Ensino/aprendizagem apropriado ao perfil & 4 & 2 & 0 & 3 \\
\hline Facilidade de auto-avaliação & 3 & 2 & 0 & 4 \\
\hline Facilidade de busca de adversário & 2 & 1 & 3 & 4 \\
\hline Tirar dúvidas & 3 & 2 & 2 & 4 \\
\hline Formação de grupos & 2 & 0 & 3 & 4 \\
\hline Registro das interações & 2 & 2 & 2 & 4 \\
\hline Facilidade de organizar eventos & 3 & 0 & 2 & 4 \\
\hline Facilidade de visualizar eventos no ambiente & 2 & 0 & 3 & 4 \\
\hline
\end{tabular}

Analisando-se as tabelas 1 e 2, podemos dividir os ambientes em dois grupos: a abordagem tradicional (I) e as abordagens baseadas em computadores (II, III e IV). A tabela 1 mostra que a abordagem IV é a que possui maior riqueza de interações, propiciando interações do tipo Pessoa-Pessoa, Pessoa-Agente, Agente-Pessoa e AgenteAgente.

Analisando-se a tabela 2 observa-se que dentre as abordagens baseadas em computadores, a abordagem IV (coluna IV) é dominante sobre as demais abordagens (II e III). Comparando-se, agora, a abordagem do AVAX (IV) com a abordagem tradicional (I), despontam fatores a favor da abordagem IV tais como: a facilidade de buscar adversários apropriados ao seu nível de jogo, a facilidade de auto-avaliação e o registro automatizado das interações.

\section{Conclusões e Trabalhos Futuros}

Neste artigo apresentou-se o AVAX, seguindo a arquitetura proposta por Netto et al. (Netto et al., 2004). A avaliação da versão atual sugere uma melhora qualitativa, quando comparada com os softwares tradicionais usados em treinamento de Xadrez (Chessmaster, ICC, entre outros), pois ao se usar agentes interagindo com pessoas e com outros agentes, as interações tornam-se, e em particular a aprendizagem, mais sintonizada com o perfil do usuário. O uso de agentes de naturezas diversas (pessoas e softwares) amplia as oportunidades de aprendizagem e de socialização entre os participantes.

A próxima etapa do projeto é a criação de novos agentes, que implementam os demais serviços específicos do Xadrez ainda não programados e suas integrações ao ambiente. Serão criados também agentes responsáveis pela gerência de grupos. Estes novos agentes integrarão a versão para Web do AVAX. Após esta etapa o AVAX será utilizado durante o $2^{\circ}$ Semestre de 2005 em eventos de aprendizagem por pequenos grupos de estudantes.

\section{Referências Bibliográficas}

AmCorA. Ambiente Cooperativo de Aprendizagem. Disponível em <http://www.gaia.ufes.br/ amcora> . Acesso em Março de 2005.

BULL, S.; GREER, J.; McCALLA, G.; KETTEL, L.; BOWES, J. User Modelling in I-Help: What, Why, When and How. In: User Modeling 2001: 8th International Conference. Springer-Verlag, Berlin Heidelberg. Disponível em $<$ http://www.eee.bham.ac.uk/bull/ papers/ UM01.html>. Acesso em Março de 2005.

CHESSMASTER. Chessmaster 10000. Disponível em <http://chessmaster.ubi.com $>$. Acesso em Janeiro de 2005. 
DETERS, R. Developing and Deploying a Multi Agent System. Disponível em $<$ http://delivery.acm.org >. Acesso em Março 2005.

DIRENE, A.; SCHAFER, H. Conceitos e Ferramentas para Apoiar o Ensino de Xadrez através de Computadores. XI Simpósio Brasileiro de Informática na Educação. Maceió: Sociedade Brasileira de Computação. (2000).

DIRENE, A.; BONA, L.C.E.; SILVA, F.; SANTOS, G.; GUEDES, A.L.P.; CASTILHO, M.A.; SUNYE, M.S.; HARTMANN, C.M. ; ANDRADE NETO, P.R.; MELLO, S. Conceitos e Ferramentas de Apoio ao Ensino de Xadrez nas Escolas Brasileiras. X Workshop sobre Informática na Escola. Salvador: Sociedade Brasileira de Computação. (2004).

FIPA. Foundation for Intelligent Physical Agents. Disponível em $<$ http://www.fipa.org >. Acesso em Março de 2005.

ICC. Internet Chess Club. Disponível em $<$ http://www.chessclub.com/ $>$. Acesso em Junho de 2005.

JADE. Java Agent Development Framework. Disponível em <http://jade.tilab.com>. Acesso em Março de 2005.

JSP. JavaServer Pages. Disponível em <http://java.sun.com/products/jsp/>. Acesso em Março de 2005.

J2SE. Java 2 Platform, Standard Edition (J2SE). Disponível em $<$ http://java.sun.com/j2se/>. Acesso em Março de 2005.

MARKHAM, S. Applying Agent Technology to Evaluation Tasks in E-Learning Environments. Disponível em <http://www.monash.edu.au/groups/flt/eet/abstracts/>. Acesso em Março de 2005.

MENEZES, Crediné Silva.; CAMPOS, Gilda Helena; CURY, Davidson. AmCorA: um Ambiente Cooperativo para a Aprendizagem Construtivista Utilizando a Internet. X Simpósio Brasileiro de Informática na Educação. Curitiba: Sociedade Brasileira de Computação. (1999).

MENEZES, Crediné Silva; VESCOVI-NETTO, Hylson; PESSOA, José Marques. AmCorA: uma Experiência com Construção e Uso de Ambientes Virtuais no Ensino Superior. XIV Simpósio Brasileiro de Informática na Educação. Rio de Janeiro: Sociedade Brasileira de Computação. (2003).

MySQL. MySQL. Disponível < http://www.mysql.com>. Acesso em Fevereiro de 2005.

NETTO, José Francisco Magalhães. Um Tutor Inteligente para o Ensino de Xadrez. Rio de Janeiro: COPPE/ UFRJ. Tese de Mestrado.

NETTO, José Francisco Magalhães; MENEZES, Crediné Silva; CASTRO, Alberto. AmCorA: Uma Arquitetura Multiagente Baseada em FIPA. XV Simpósio Brasileiro de Informática na Educação, Manaus: Sociedade Brasileira de Computação. (2004). 
NETTO, José Francisco Magalhães; TAVARES, Orivaldo; MENEZES, Crediné Silva. AVAX - Ambiente Virtual para Aprendizagem de Xadrez. Simpósio Brasileiro de Jogos para Computador e Entretenimento Digital (SBGames 2005). São Paulo: Sociedade Brasileira de Computação. (2005a).

NETTO, José Francisco Magalhães; TAVARES, Orivaldo; MENEZES, Crediné Silva. Ambiente Virtual para Aprendizagem de Xadrez. Workshop de Jogos Educacionais. XVI Simpósio Brasileiro de Informática na Educação. Juiz de Fora: Sociedade Brasileira de Computação. (2005b).

SILVEIRA, Ricardo Azambuja; VICARI, Rosa Maria. Improving Interactivity in ELearning with JADE - Java Agent Framework for Distance Learning Environments. International Conference on Engineering Education. Manchester: (2002).

VASSILEVA, J.; DETERS, R.; GREER, J.; McCALLA, G.; BULL, S.; KETTEL, L. Lessons from Deploying I-Help. Disponível em $<$ http://julita.usask.ca/mable/vassileva.pdf $>$. Acesso em Março 2004.

VASSILEVA, J. Motivating Participation in Virtual Learning Communities. Disponível em: <http://julita.usask.ca/Texte/ICWES12.pdf > . Acesso em Março de 2004.

VIGOTSKY, Lev. A Formação Social da Mente. São Paulo: Livraria Martins Fontes, 1998.

WOOLDRIDGE, M. An Introduction to MultiAgent Systems. Inglaterra: Editora John Wiley\&Sons, 2002. 